\title{
Internet of Personalized and Autonomous Things (IoPAT)
}

\author{
Smart Homes Case Study
}

\author{
Salma Elmalaki \\ University of California, Los Angeles \\ selmalaki@ucla.edu
}

\author{
Yasser Shoukry \\ University of Maryland, College Park \\ yshoukry@ece.umd.edu
}

\author{
Mani Srivastava \\ University of California, Los Angeles \\ mbs@ucla.edu
}

\begin{abstract}
IoT devices are permeating every corner of our lives today paving the road for more substantial smart systems. Despite their ability to collect and analyze a significant amount of sensory data, traditional IoT typically depends on fixed policies and schedules to enhance user experience. However, fixed policies that do not account for variations in human mood, reactions, and expectations, fail to achieve the promised user experience. In this paper, we propose an architecture for personalized and autonomous IoT systems that weaves personalization and context-awareness into the very fabric of smart systems. By building upon ideas from reinforcement learning, we show-using an example of smart and personalized home services-how the proposed architecture can adapt to human behaviors that are varying between individuals and vary, for the same individual, across time while addressing some of the security and privacy challenges.
\end{abstract}

\section{CCS CONCEPTS}

- Human-centered computing $\rightarrow$ Contextual design; Ubiquitous computing;

\section{KEYWORDS}

Personalized IoT, Autonomous Systems, Smart Homes

\section{ACM Reference Format:}

Salma Elmalaki, Yasser Shoukry, and Mani Srivastava. 2018. Internet of Personalized and Autonomous Things (IoPAT) : Smart Homes Case Study. In The 1st ACM International Workshop on Smart Cities and Fog Computing (CitiFog'18), November 4, 2018, Shenzhen, China. https://doi.org/10.1145/ 3277893.3277901

\section{INTRODUCTION}

The age of autonomous IoT systems that adapt to human behaviors has arrived. In these context-aware and pervasive systems (that we term as the Internet of Personalized and Autonomous Things or IoPAT for short), human reactions and behaviors are observed by edge devices and are used to adapt the behavior of the whole IoT. By continuously developing a cognition about the environment and the human state, and adapting accordingly, IoPAT can provide the user with a unique and personalized experience. IoT

Permission to make digital or hard copies of all or part of this work for personal or classroom use is granted without fee provided that copies are not made or distributed for profit or commercial advantage and that copies bear this notice and the full citation on the first page. Copyrights for components of this work owned by others than ACM must be honored. Abstracting with credit is permitted. To copy otherwise, or republish, to post on servers or to redistribute to lists, requires prior specific permission and/or a fee. Request permissions from permissions@acm.org.

CitiFog'18, November 4, 2018, Shenzhen, China

(C) 2018 Association for Computing Machinery.

ACM ISBN 978-1-4503-6051-7/18/11 . \$ \$15.00

https://doi.org/10.1145/3277893.3277901 systems can enable a multitude of applications in the context of smart buildings [2], smart cities [38, 40], healthcare [22, 29, 32], and automotive systems [11] by providing services that are not only adaptable to the human but can also intervene and maybe override human actions to ensure his safety and satisfaction. While traditional IoT systems interact with humans, in general, by collecting data directly from humans and their environment, a unique feature of IoPAT is its ability to assess human satisfaction and closing the loop by taking actions to adapt to the changes in his mood, needs, and expectations. This tight coupling between human behavior and computing promises a radical change in human life [31]. To emphasize the difference between the proposed IoPAT and traditional IoT, we consider the example of smart thermostats in the context of smart homes. Current state-of-art smart thermostats are capable of adapting the home temperature based on room occupation [4] using fixed schedules and policies [26]. For example, homeowners are required to define a preset of configurations, and the IoT system makes sure to follow these configurations. Unfortunately, human needs vary across time. In the context of smart home, since body's temperature needs to drop to sleep [20] and since body's temperature is affected by multiple factors like excitement, anxiety, body activity, and health issues, the same human may prefer a cooler or warmer temperature during sleep time. Even more, in the same mood, health, and activity situations, the same person may have a different preference for the best room temperature. Unfortunately, due to this variation in human needs and behavior, current IoT are heteronomous and incapable of providing the necessary level of personalization. Thanks to the recent advances in computational power, memory capacities [16], networking bandwidths, and battery capabilities [10], IoT devices are now capable of performing machine learning algorithms to infer complex human states and adapt to them [12, 28, 33, 35]. However, very few works in literature focused on assessing personalized needs and satisfaction to adapt to complex human states that vary between individuals and vary for the same individual across time [11]. It is these challenges in building personalized and autonomous systems that we study in this paper.

\section{IOPAT SYSTEMS}

In IoPAT systems, the first step is extracting complex semantics from various sensory data to infer the state (or context) of both the human users along with their physical environment. Such information can be collected from various edge devices including thermostat sensors, mobile phones, and wearables. These raw sensory data can be used to infer complex human states including human activity (e.g., exercising, running, walking) [24] and mood [25]. Thanks to the increasing computational capabilities at the edge devices and sophisticated machine learning models, such state and context inferences become ubiquitous. 


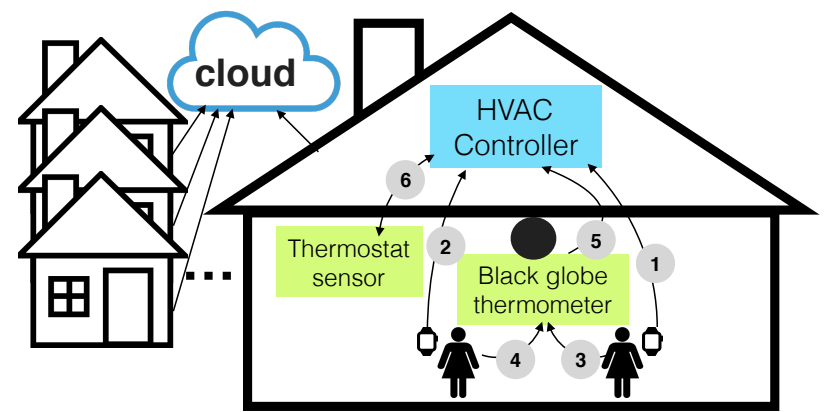

Figure 1: Smart Home with smart HVAC controller. Wearable devices number (1) and (2) at the edge reports the human activity (sleeping, standing, resting,...etc), the black globe thermometer sensor number (3) and (4) estimates the mean radiant temperature which affects the thermal comfort of the human, while (5) reports the thermal comfort to the HVAC controller (6) sets/reports the indoor temperature to the controller.

The next step is to fuse the individual states inferred from various edge devices along with those relayed from the cloud. This global state is then utilized by adaptation algorithms to close the loop and take actions in an attempt to match the user and environment state. Unfortunately, the human preferences vary between different humans and even for the same human across time. Therefore, a unique aspect of IoPAT is their ability to assess the human satisfaction and "learn" how to correct their actions to enhance the user experience.

To better illustrate the potential of IoPAT systems over traditional IoT, we consider the example of a smart home shown in Figure 1. Current state-of-the-art smart IoT-based thermostats are capable of regulating home temperature based on weather forecasts and occupancy [8]. That is, these smart thermostats can respond to real-time changes in the outdoor temperature based on the number of the occupants of the home. However, these systems ignore a fundamental fact. Human comfort temperature varies across individuals and, even more, it varies across time for the same individual. A home occupant may prefer a particular home temperature while reading and prefer another temperature while working out. Even for the same activity (e.g., reading), the same individual may prefer different temperatures across time. This lack of adapting to human variations in human preferences and responses is the major drawback in the current IoT systems.

To circumvent this performance gap and provide every human with a personalized experience, the proposed IoPAT in Figure 1 utilizes sensor information available at different edge devices like mobile phone and wearable sensors to continuously infer complex human states (e.g., activity and mood). Upon changes in any of these states, the IoPAT-based thermostat utilizes the history of this particular individual to take an action and change the home temperature. However, as discussed before, individual humans may change their comfort zone, the IoPAT-based thermostat monitors the comfort of the user using a black-globe thermometer and readapt accordingly. That is, the IoPAT system needs to continuously "learn" the new human preference to close the loop and provide a unique experience for each human.

\subsection{Challenges}

We conclude this section by highlighting several challenges that arise in the context of IoPAT. In addition to the traditional challenges in IoT which include power, memory, connectivity, and computational issues, IoPAT faces the following challenges:

\subsubsection{Adaptability.}

As argued in the smart home example, IoPAT system needs to continuously monitor the satisfaction of the human user, and "learn" and "correct" the actions accordingly. The two types of variations namely (i) variations between individuals and (ii) variations within the same individual [11] are central themes in IoPAT that need to be carefully addressed.

After IoPAT systems adapt to human by deciding different actions, human users will respond to these actions. Similarly to the variations in human preferences, is the variations in the time for a human to respond to the actions taken by IoPAT. While some users may react to every action taken by IoPAT, others may react after an IoPAT has already performed multiple actions. To correctly learn the human preference, IoPAT systems must take into account this variation in the observed human response time.

A related challenge is concerned with the ability to fuse complex human states observed from different individuals. Thanks to the current advances in machine learning, several works in the literature reported significant breakthroughs in observing individual human states $[9,18,30]$. However, very few works focused on the problem of fusing these individual human states to build complex states that represent the aggregate behavior of all humans interacting with the system [39].

\subsubsection{Privacy.}

Unfortunately, the same act of adapting to the human context often leads to systems where increased sophistication comes at the expense of more privacy weaknesses. At the heart of IoPAT privacy is the notion that actions taken by IoPAT-in according to information collected about human's state-poses a significant privacy risk on inferring user sensitive information. By simply monitoring the patterns of the actions taken by an IoPAT, an adversarial eavesdropper may be able to reverse engineer these patterns to leak information about the human state.

Consider the smart home example discussed in the previous subsection. To maximize the user experience, the IoPAT-based smart home changes the home temperature to match the human mood. That is, to compensate for rising skin temperature during excitement, the smart home will lower the home temperature and vice versa. Now consider a spyware installed on the HVAC actuator. By monitoring the actions decided by the IoPAT system, this spyware can infer the human mood without having access to the raw data collected by the sensors on the other edge devices. In general, current machine learning algorithms are adequate to discover repetitive patterns which can be related back to different human states.

Detecting the amount of information that IoPAT actions could leak is a challenging problem. Unlike traditional spyware where a priori information about its code signature or input-output behavior is known, this privacy leaking spyware is not identified yet. This opens the question of how to design a generic detection and 


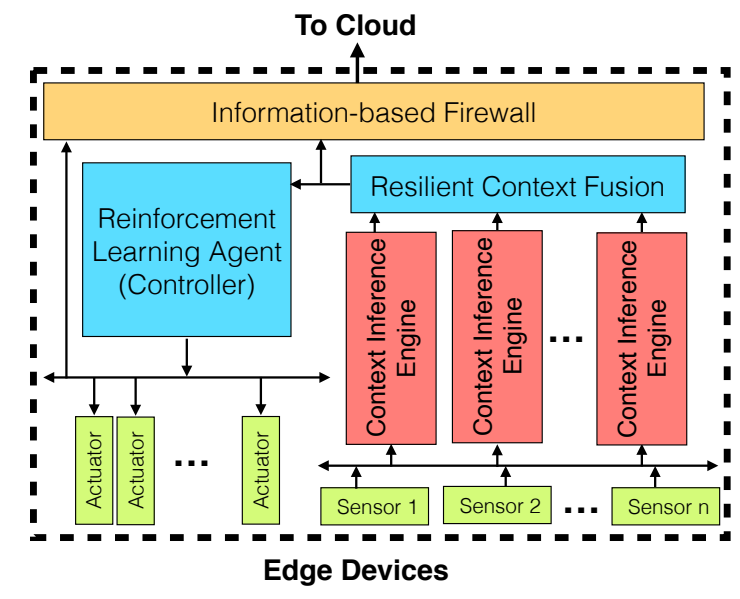

Figure 2: Proposed architecture for IoPAT.

mitigation algorithms that can limit the amount of information that could be leaked using such spyware.

\subsubsection{Security.}

While IoPAT provides a unique opportunity to empower humans and fit their needs, they are susceptible to a unique threat vector; the human himself. That is, the humans interacting with these devices are not always collaborative agents; they can be malicious entities who take an adversary role in the interaction with IoPAT systems. For instance, Microsoft Twitter chatbot, Tay [27], is an infamous example of how artificial intelligence agents can be fooled by humans to perform unexpectedly.

In the context of the smart home example discussed before, a malicious human may intentionally behave in a way that fools the machine learning algorithms that estimates his state in an attempt to force the HVAC controller taking incorrect actions leading to liability issues. This opens the question of how to design resilient policies against malicious humans.

\section{IOPAT ARCHITECTURE}

In this section, we propose an architecture of IoPAT system that addresses some of the challenges mentioned in the previous section. As shown in Figure 2, our architecture for IoPAT consists of three main subsystems. Below we give more details about each of these subsystems.

\subsection{Resilient Context Fusion}

The first step in an IoPAT system is to utilize the available sensors to infer the state of the human and his environment. Such inference can take place directly from multiple raw sensor measurements or by fusing different low level inferred states. While context inferences and context adaptation frameworks have been studied thoroughly in the literature $[9,12,18,30]$, as motivated in Section 2.1.3, IoPAT is susceptible to a unique threat vector namely the human himself. An individual human may try to maliciously influence the inference engine in an attempt to lead the IoPAT system to decide wrong actions which may trigger several liability concerns. To alleviate this challenge, a commonly explored idea is to exploit the redundancy in the collected sensor data in order to identify and isolate the malicious data leading to a new set of resilient learning-based context inference and fusion algorithms [37].

\subsection{Reinforcement Learning Controller}

Once the human and the environment state (or context) are determined, this state is then used to close the loop and take adaptation actions. As mentioned in Section 2.1.1, adapting to variations between humans and variations within the same human are the central challenge in designing an IoPAT system. To address these challenges, the IoPAT controller continuously monitors the human reactions. If the human dislikes the actions taken by the controller (as reflected by changes in his state), then the IoPAT controller "learns" the human preference otherwise the controller "learns" that this action matches the human preference. This continuous feedback loop of taking actions and monitoring the human decision (response) fits naturally within the Reinforcement Learning (RL) paradigm. In the RL problem, a software agent tries to learn the behavior of an environment by issuing actions and observing the change in the state of the environment with the purpose of maximizing a notion of total reward. To model variations between the state of the individuals and the variation within the same individual, we model the human as a Markov Decision Process (MDP), where the states in the MDP correspond to the human state. Each action by the IoPAT controller will lead to a transition in this MDP. However, the transition probabilities between these states are unknown in advance due to the intrinsic variation in the human preference. In order to solve the MDP to get the best action for each state without knowing the transitional probabilities, our IoPAT controller is equipped with an RL algorithm, named Q-learning algorithm, that best fits this problem setup. In the Q-learning algorithm, an agent applies an action on the environment and observes its effect per state. The agent chooses an action which maximizes a notion of reward value. The reward value is a quantification of how good the taken action by the agent.

\subsection{Information-Based Firewall}

Sharing information in the context of IoPAT systems may lead to privacy leaks stemming from the tight coupling between human behavior and actions produced by IoPAT controllers. Spyware exploiting these privacy leaks cannot be detected by the current state-of-the-art signature-based and behavior-based detection techniques. Our architecture uses an information-based detection and mitigation firewall that aims at breaking the correlation between the human context and the controller actions.

\section{SMART HOME CASE STUDY}

In this section, we study the effect of the IoPAT architecture proposed in Section 3 using a smart home example. In particular, for the purpose of this paper, we only focus on the proposed RL-based controller. Recent work in literature targets personalized HVAC [21], while trying to assist the human satisfaction [19] with fairness across all occupants [36]. In IoPAT, we target personalization of thermal house while taking into account the change in human preferences across time. 


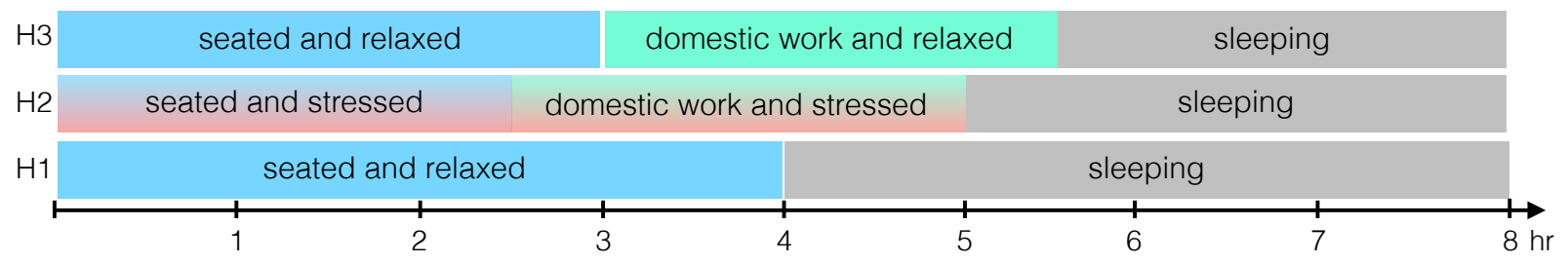

Figure 3: Timeline of the activity for the three humans.

To that end, we conducted numerical simulation using the thermal model of a house with three occupants. We simulated a period of 8 hours in total with a sample time of 30 second. We implemented the proposed IoPAT RL-based controller and a traditional IoT-based thermostat. Details about the high-fidelity mathematical model for the house, humans, and the IoPAT controller are given below.

\subsection{Thermal Model of a House}

In our simulations, we utilize a thermodynamic model of the house that takes into consideration the geometry of the house, the number of windows, the roof pitch angle, and the type of the insulation used. The house is being heated by a heater with a flow of air with temperature $50^{\circ} \mathrm{c}$. A thermostat is used to allow a fluctuation of $2.5^{\circ} \mathrm{c}$ above and below the desired set-point which specifies the temperature that must be maintained indoors. We consider two external controllers that set the desired set-point. A classical IoT thermostat that determines the set-point based on the number of occupants in the house. The second controller is the IoPAT controller explained in Section 4.3.

To take into account the personal behavior, we model each human as a heat source with heat flow that depends on the average exhale breath temperature (EBT) and the respiratory minute volume (RMV) [17]. These two parameters are highly dependent on the human activity. For example, $R M V \approx 61 / \mathrm{m}$ when the human is resting while $R M V \approx 12 \mathrm{l} / \mathrm{m}$ represents a human performing moderate exercise [6].

\subsection{Human Thermal Comfort}

We use the Prediction Mean Vote (PMV) [15] as a measure for the human thermal comfort. PMV gives a score on how warm/cold a person feels. It depends mainly on the human activity, metabolic rate, clothing, and environmental variables (air speed, air temperature, mean radiant temperature, and vapor pressure of air).

The PMV score ranges from -3 to 3 which is the range of thermal sensation from very cold (-3) to very hot (3). According to ISO standard ASHRAE 55 [3], a PMV in the range of -0.5 and +0.5 for an interior space is recommended to achieve thermal comfort. PMV score for each human can be estimated using the knowledge of the clothing factor, the clothing insulation, the metabolic rate, the air temperature, the air vapor pressure, and the mean radiant temperature [15].

In our IoPAT controller, we assume the knowledge of the weather forecast which affects the clothing factor and clothing insulations.
For example, in our experiments, we assume a winter season and hence the IoPAT controller fixes an average for the clothing insulation of 0.9 clo [3]. The metabolic rate is determined by the occupant activity [13] and stress level (relaxed/stressed) [34]. This can be inferred using the heart rate and respiration rate either using wearable sensors [7] or using non-invasive technologies [1]. Air temperature can be directly measured using thermostat sensors, while air vapor pressure is a one-to-one correspondent to the ambient temperature [14] which can be directly measured using outside thermostat as well. Finally, the mean radiant temperature can be estimated using a black-globe thermometer $[5,23]$.

\subsection{RL-based Controller for IoPAT}

While human stress level (relaxed/stressed) and human activity (resting, watching TV, eating, ...) can affect their thermal comfort, the IoPAT RL-based controller needs to track their PMV score and take correcting actions to maximize their comfort. Accordingly, we model each human as an MDP. States in the MDP corresponds to different PMV scores (with 0.5 granularity) resulting in 14 states for each human. We design the reward function for the RL-based controller on the difference between the average PMV (across all humans) and the nominal value -0.5 to 0.5 . The RL-based controller then changes the temperature set-point accordingly. While the relation between the actions (changes in the set-point) and the human comfort is unknown to the controller, we use a Q-learning algorithm to continuously "learn" the human response to different set-points and take corrective actions.

The timeline for the activity of the three occupants can be summarized in Figure 3.

\subsection{Numerical Results}

Using the simulation environment discussed in Sections 4.1 and 4.2, we consider the scenario in which the state of the three occupants change over time and report the thermal comfort for each human when both the IoT based controller (which changes the set-point according to the occupancy and weather forecast) and the proposed IoPAT controller are used to controlling the house temperature. During the simulation time, we change the state of the three occupants. We summarize the scenarios in our simulation as follows:

- Human 1: is sitting and relaxed for the first 4 hours. He then goes to sleep for the remaining 4 hours. 

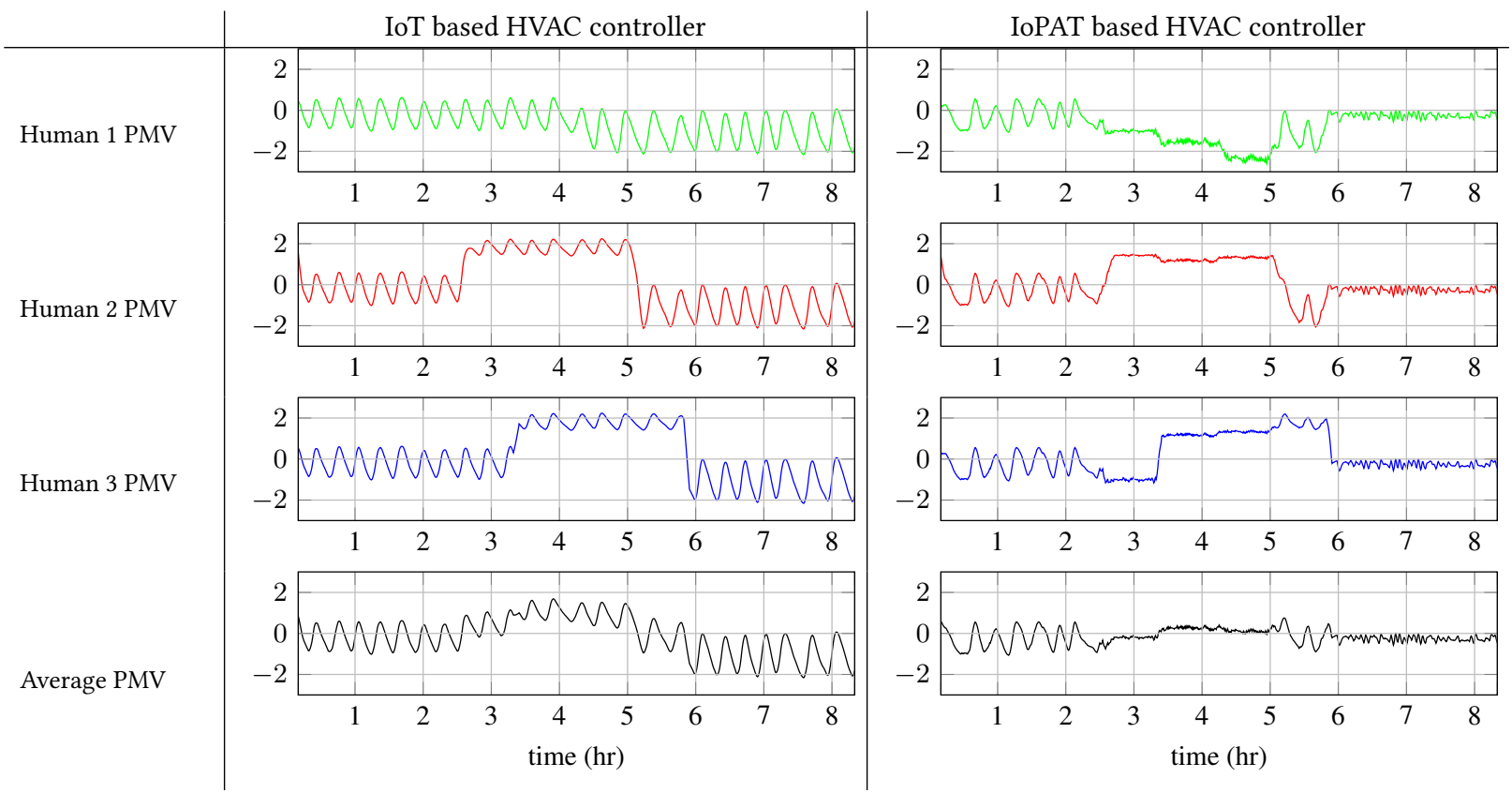

Figure 4: Prediction Mean Vote (PMV) for the three occupants ( $y$-axis) across time ( $x$-axis) using IoT system (left) and IoPAT system (right) for varying occupants' activity and stress level (relaxed/stress).

- Human 2: is sitting and stressed for 2.5 hours, performing domestic work while still being stressed for another 2.5 hours, and finally going to sleep for the remaining 3 hours.

- Human 3: is sitting and relaxed for 3 hours, performing domestic work for 2.5 hours while still being relaxed, and then finally going to sleep for 2.5 hours.

Figure 4 reports the comparison between the IoT controller and the IoPAT controller with respect to the individual thermal comfort and the average thermal comfort. During the first 2.5 hours, when the three occupants are seated, the IoT controller shows a good range of PMV (between -0.5 to 0.5 ) at a set-point of $23^{\circ} \mathrm{c}$. Similarly, the RL-based controller chooses an action of setting the set-point at $23^{\circ} \mathrm{c}$.

However, once human 2 and human 3 start to perform domestic work, their average exhale breath temperature increases which affects their thermal comfort. While the IoT controller is only affected by the occupancy and the air temperature, it decides to still provide a set-point of $23^{\circ} \mathrm{c}$ leading to a higher PMV, almost reaching at value 2. In contrast, thanks to the feedback from the different sensors, the reward for the RL-based controller starts to decrease forcing the RL-based controller to take corrective actions. Accordingly, the RL-based controller starts to gradually decrease the set-point to $21^{\circ} \mathrm{c}$ followed by a further decrease to $20^{\circ} \mathrm{c}$ to compensate the rise in PMV for human 2 and human 3 leading to a decrease in the individual and average PMV.

Similarly, in the last 3 hours, all occupants are sleeping and hence their average exhale breath drops and their comfort score also decreases. Again, the IoT controller uses the occupancy based policy leading to the same set-point of $23^{\circ} \mathrm{c}$ which in turn leads to a drop in the PMV score reaching a value of -2 . On the other side, and thanks again to the RL-based controller, it decides to increase the set-point gradually until it reaches $25^{\circ} \mathrm{c}$ to compensate the decrease in the PMV.

These results show the effectiveness of the proposed RL-based controller for IoPAT systems to track the variations in the human behavior and provide a personalized experience as compared to traditional IoT systems. However, it comes with the cost of a $2 \%$ increase in energy consumption at the end of the simulated 8 hours. This is stemmed from the fact that the heater had to operate more in case of IoPAT to raise the temperature when the three occupants were sleeping.

\subsection{Execution Time}

We report the execution time of the RL-based controller running on $2.6 \mathrm{GHz}$ Intel Core $i 5$ with 8 GB RAM. The state observation (and inference) routine to detect the state of the human consumes $20.4 \mathrm{~ms}$, while the feedback loop in the RL-agent (using Q-leaning) to learn the best action for the current state consumes $104 \mathrm{~ms}$.

\section{CONCLUSION}

Our everyday life activities are becoming more dependent on IoT devices. Since most of these devices interact with humans, the IoT system should include the human factor into the loop of computation. Accordingly, we propose IoPAT system. IoPAT considers the human preference and current state as an integral part to the IoT system. In this paper, we proposed an architecture for IoPAT and discussed the challenges that face the personalization of IoT. We showed a case study of a smart house using IoPAT, in which we compared to the regular IoT with respect to the thermal comfort of the occupants. 


\section{ACKNOWLEDGMENTS}

This research was supported in part by the U.S. Army Research Laboratory under Cooperative Agreement W911NF-17-2-0196, by the National Science Foundation under award \# OAC-1640813 and IIS-1636916, and the King Abdullah University of Science and Technology (KAUST) through its Sensor Innovation research program. The Microsoft Research PhD Fellowship has supported Salma Elmalaki. Any findings in this material are those of the author(s) and do not reflect the views of any of the above funding agencies. The U.S. Government is authorized to reproduce and distribute reprints for Government purposes notwithstanding any copyright notation hereon.

\section{REFERENCES}

[1] Fadel Adib, Hongzi Mao, Zachary Kabelac, Dina Katabi, and Robert C Miller. 2015 Smart homes that monitor breathing and heart rate. In Proceedings of the 33rd annual ACM conference on human factors in computing systems. ACM, 837-846.

[2] Yuvraj Agarwal, Bharathan Balaji, Rajesh Gupta, Jacob Lyles, Michael Wei, and Thomas Weng. 2010. Occupancy-driven energy management for smart building automation. In Proceedings of the 2nd ACM workshop on embedded sensing systems for energy-efficiency in building. ACM, 1-6.

[3] ASHRAE/ANSI Standard 55-2010 American Society of Heating, Refrigerating, and Air-Conditioning Engineers. 2010. Thermal environmental conditions for human occupancy. Inc.Atlanta, GA, USA (2010).

[4] Bharathan Balaji, Jian Xu, Anthony Nwokafor, Rajesh Gupta, and Yuvraj Agarwal. 2013. Sentinel: Occupancy Based HVAC Actuation Using Existing WiFi Infrastructure Within Commercial Buildings. In Proceedings of the 11th ACM Conference on Embedded Networked Sensor Systems (SenSys '13). 17:1-17:14

[5] Th Bedford and CG Warner. 1934. The globe thermometer in studies of heating and ventilation. Epidemiology \& Infection 34, 4 (1934), 458-473.

[6] Robert G. Carroll. 2007. Pulmonary System. In Elsevier's Integrated Physiology. Elsevier, Chapter 10, 99-115.

[7] Jongyoon Choi and Ricardo Gutierrez-Osuna. 2009. Using heart rate monitors to detect mental stress. In Wearable and Implantable Body Sensor Networks, 2009. BSN 2009. Sixth International Workshop on. IEEE, 219-223.

[8] Adrian K Clear, Janine Morley, Mike Hazas, Adrian Friday, and Oliver Bates. 2013. Understanding adaptive thermal comfort: new directions for UbiComp. In Proceedings of the 2013 ACM international joint conference on Pervasive and ubiquitous computing. ACM, 113-122.

[9] Diane J Cook, Maureen Schmitter-Edgecombe, and Prafulla Dawadi. 2015. Analyzing activity behavior and movement in a naturalistic environment using smart home techniques. IEEE journal of biomedical and health informatics 19, 6 (2015), 1882-1892.

[10] Salma Elmalaki, Mark Gottscho, Puneet Gupta, and Mani B Srivastava. 2014 A Case for Battery Charging-Aware Power Management and Deferrable Task Scheduling in Smartphones.. In HotPower.

[11] Salma Elmalaki, Huey-Ru Tsai, and Mani Srivastava. 2018. Sentio: Driver-in-theLoop Forward Collision Warning Using Multisample Reinforcement Learning. In Proceedings of the 16th annual ACM conference on Embedded Networked Sensor Systems. ACM.

[12] Salma Elmalaki, Lucas Wanner, and Mani Srivastava. 2015. Caredroid: Adaptation framework for android context-aware applications. In Proceedings of the 21st Annual International Conference on Mobile Computing and Networking. ACM, 386-399.

[13] Engineering ToolBox. 2004. "Metabolic Rate". https://www.engineeringtoolbox. com/met-metabolic-rate-d_733.html. (2004)

[14] Engineering ToolBox. 2004. "Relative Humidity in Air". https://www. engineeringtoolbox.com/relative-humidity-air-d_687.html. (2004).

[15] Poul O Fanger. 1970. Thermal comfort. Analysis and applications in environmental engineering. Thermal comfort. Analysis and applications in environmental engineering. (1970).

[16] Mark Gottscho, Irina Alam, Clayton Schoeny, Lara Dolecek, and Puneet Gupta. 2017. Low-Cost Memory Fault Tolerance for IoT Devices. (2017).

[17] Jitendra K Gupta, Chao-Hsin Lin, and Qingyan Chen. 2010. Characterizing exhaled airflow from breathing and talking. Indoor air 20, 1 (2010), 31-39.

[18] Kun Han, Dong Yu, and Ivan Tashev. 2014. Speech emotion recognition using deep neural network and extreme learning machine. In Fifteenth Annual Conference of the International Speech Communication Association.

[19] Lam Abraham Hang-yat and Dan Wang. 2013. Carrying my environment with me: A participatory-sensing approach to enhance thermal comfort. In Proceedings of the 5th ACM Workshop on Embedded Systems For Energy-Efficient Buildings. ACM, 1-8.
[20] Harvard Medical School. 2007. "The Characteristics of Sleep." http://healthysleep. med.harvard.edu/healthy/science/what/characteristics. (2007).

[21] Wooyoung Jung and Farrokh Jazizadeh. 2017. Towards integration of doppler radar sensors into personalized thermoregulation-based control of HVAC. In Proceedings of the 4th ACM International Conference on Systems for Energy-Efficient Built Environments. ACM, 21.

[22] MPR Sai Kiran, Pachamuthu Rajalakshmi, Krishna Bharadwaj, and Amit Acharyya. 2014. Adaptive rule engine based IoT enabled remote health care data acquisition and smart transmission system. In Internet of Things (WF-IoT), 2014 IEEE World Forum on. IEEE, 253-258.

[23] LA Kuehn, RA Stubbs, and RS Weaver. 1970. Theory of the globe thermometer. fournal of applied physiology 29, 5 (1970), 750-757.

[24] Oscar D Lara, Alfredo J Pérez, Miguel A Labrador, and José D Posada. 2012. Centinela: A human activity recognition system based on acceleration and vital sign data. Pervasive and mobile computing 8, 5 (2012), 717-729.

[25] Robert LiKamWa, Yunxin Liu, Nicholas D Lane, and Lin Zhong. 2013. Moodscope: Building a mood sensor from smartphone usage patterns. In Proceeding of the 11 th annual international conference on Mobile systems, applications, and services. ACM, 389-402.

[26] Jiakang Lu, Tamim Sookoor, Vijay Srinivasan, Ge Gao, Brian Holben, John Stankovic, Eric Field, and Kamin Whitehouse. 2010. The smart thermostat: using occupancy sensors to save energy in homes. In Proceedings of the 8th ACM Conference on Embedded Networked Sensor Systems. ACM, 211-224.

[27] Microsoft. 2015. “Tay.ai”. https://twitter.com/tayandyou. (2015).

[28] Subhas Chandra Mukhopadhyay. 2015. Wearable sensors for human activity monitoring: A review. IEEE sensors journal 15, 3 (2015), 1321-1330.

[29] Anh Nguyen, Raghda Alqurashi, Zohreh Raghebi, Farnoush Banaei-Kashani, Ann C Halbower, and Tam Vu. 2016. A lightweight and inexpensive in-ear sensing system for automatic whole-night sleep stage monitoring. In Proceedings of the 14th ACM Conference on Embedded Network Sensor Systems CD-ROM. ACM, 230-244.

[30] Olukunle Ojetola, Elena I Gaura, and James Brusey. 2011. Fall detection with wearable sensors-safe (Smart Fall Detection). In Seventh International Conference on Intelligent Environments (IE), 2011. IEEE, 318-321.

[31] Rosalind W Picard. 1995. Affective Computing-MIT Media Laboratory Perceptual Computing Section Technical Report No. 321. Cambridge, MA 2139 (1995)

[32] Darius Adam Rohani, Helge BD Sorensen, and Sadasivan Puthusserypady. 2014. Brain-computer interface using P300 and virtual reality: a gaming approach for treating ADHD. In Engineering in Medicine and Biology Society (EMBC), 2014 36th Annual International Conference of the IEEE. IEEE, 3606-3609.

[33] Akane Sano and Rosalind W Picard. 2013. Stress recognition using wearable sensors and mobile phones. In Humaine Association Conference on Affective Computing and Intelligent Interaction (ACII). IEEE, 671-676.

[34] G Seematter, C Binnert, and L Tappy. 2005. Stress and metabolism. Metabolic syndrome and related disorders journal 3, 1 (2005), 8-13.

[35] Tal Shany, Stephen J Redmond, Michael R Narayanan, and Nigel H Lovell. 2012. Sensors-based wearable systems for monitoring of human movement and falls. IEEE Sensors fournal 12, 3 (2012), 658-670.

[36] Eun-Jeong Shin, Roberto Yus, Sharad Mehrotra, and Nalini Venkatasubramanian. 2017. Exploring Fairness in Participatory Thermal Comfort Control in Smart Buildings. In Proceedings of the 4th ACM International Conference on Systems for Energy-Efficient Built Environments (BuildSys '17). ACM, 19:1-19:10.

[37] Yasser Shoukry, Michelle Chong, Masashi Wakaiki, Pierluigi Nuzzo, Alberto L. Sangiovanni-Vincentelli, Sanjit A. Seshia, João P. Hespanha, and Paulo Tabuada. 2016. SMT-based Observer Design for Cyber-physical Systems Under Sensor Attacks. (2016), 29:1-29:10.

[38] Huey-Ru Debbie Tsai, Yasser Shoukry, Min Kyung Lee, and Vasumathi Raman. 2017. Towards a socially responsible smart city: dynamic resource allocation for smarter community service. In Proceedings of the 4th ACM International Conference on Systems for Energy-Efficient Built Environments. ACM, 13.

[39] Liang Wang, Tao Gu, Xianping Tao, Hanhua Chen, and Jian Lu. 2011. Recognizing multi-user activities using wearable sensors in a smart home. Pervasive and Mobile Computing 7, 3 (2011), 287-298.

[40] Andrea Zanella, Nicola Bui, Angelo Castellani, Lorenzo Vangelista, and Michele Zorzi. 2014. Internet of things for smart cities. IEEE Internet of Things journal 1, 1 (2014), 22-32. 